\title{
Konflikt und Chance in der interkulturellen Kommunikation'
}

\author{
Dipl.-Psych. Daniel Orellana Aguirre
}

\section{Einleitung}

Zwei Fragen drängen sich mir in den Vordergrund bei diesem Thema:

- Worüber sprechen wir, wenn wir über „interkulturelle Kommunikation" sprechen?

- Wie können wir die Probleme, die sich dabei ergeben so interpretieren, daß daraus produktive bzw. schöpferische Zugänge werden?

\section{Das Problem der interkulturellen Kommunikation}

Interkulturelle Kommunikationsprobleme ergeben sich in erster Linie aus den allen Kulturen gemeinsamen Verallgemeinerungstendenzen der eigenen Deutungsschemata (Ethnozentrismus), welche in der Begegnung mit einer fremden Kultur dazu führen, diese zunächst im Sinne der eigenen auszulegen - mit der Folge der Anhäufung von Mißverständnissen. Solange dieser Zustand anhält, ergeben sich schwer zu überwindende Hindernisse für die Herausbildung gemeinsamer Handlungsstrategien und Ziele. Bereits durchgefuihrte Studien - zum Beispiel im Rahmen des internationalen Jugendaustausches - zeigen, daß Möglichkeiten der Begegnung allein nicht ausreichen, um die Annäherung von Kulturen zu gewährleisten (vgl. Haumersen/Liebe, 1989). Die Ergebnisse sprechen für sich: Die Chancen des Verstehens anderer Kulturen werden nicht genutzt, Hemmungen gegenüber Fremden werden nicht abgebaut, Gruppenkonflikte nur wenig zum gegenseitigen Lernen benutzt, die Leitungskräfte sind unsicher und überfordert, die Vorbereitungen und die Zielklarheit seitens der Träger lassen zu wünschen übrig.

\section{Das Spannungsfeld zwischen Chance und Konflikt in der interkulturellen Kommunikation}

Wir können drei Aspekte zur Analyse der Chancen und Gefahren sozialer Interaktion benutzen, die sich mit den Begriffen der Permeabilität (Durchlässigkeit oder Wandelbarkeit), der kulturellen Trägheit (Selbsterhaltungstendenz) und der Fragilität (Zerbrechlichkeit) beschreiben lassen (vgl. Mehan, Hugh \& Wood, Houston, 1976). Bei interkultureller Kommunikation treten unterschiedliche Realitätskonzepte miteinander in Beziehung. Hier liegt das Spannungsfeld zwischen gelingender und mißlingender Kommunikation von Einheimischen und Fremden. So wie Realitäten durch interkulturelle Kommuni- kation bereichert und erweitert werden, können sie auch durch zu große Diskrepanzen, z. B. ein inadäquates Tempo des Aufeinandertreffens abweichender Lebenswelten, gefährdet und zerstört werden. Ich erinnere hier an Begegnungen mit Kolonisationscharakter, die als Paradebeispiel für fehlende Interkulturalität stehen.

In der Praxis interkultureller Begegnung wird es dabei um die richtige Gestaltung der Art und Weise und der zeitlichen Abstimmung der Kommunikationsprozesse gehen. Weicht die Geschwindigkeit oder die Art und Weise der Annäherung beim Versuch der Verständigung von einem subjektiven Wert ab, ohne den drei oben erwähnten Aspekten Rechnung zu tragen, so wird in erster Linie der Aspekt der kulturellen Trägheit aktiv werden und für die Bildung von Konfliktfeldern sorgen. Jedem Deutungssystem wohnt auch die Tendenz inne, sich selbst zu erhalten, d. h. sich selbst nur dann in Frage zu stellen, wenn es keinen Zweifel mehr über seine Unbrauchbarkeit gibt. Das ist der Fall, wenn - durch die natürliche Durchlässigkeit aller Deutungssysteme - ausreichend Information in der Form von Lernen aufgenommen wurde, um eine Erweiterung zu bewirken. Vor jeder Erweiterung aber wird die bereits vertraute Deutungsform vor viel zu schnellen und wesentlichen Erweiterungen geschützt, da diese Veränderung als bedrohlich angesehen wird. Eine analoge Diskussion findet seit längerem im Zusammenhang eines Paradigmawechsels in den Naturwissenschaften statt.

Die Schutzmechanismen können von der produktiven Form einer Dialog-Herstellung mit dem Fremden bis zu pathologischen Erscheinungen wie Rassismus, Verfolgung oder Vernichtung des bedrohlichen Fremden gehen. Dabei ist zu erwähnen, daß diese natürlichen Schutzmechanismen durchaus viel zu früh und unnötigerweise aktiviert werden können, wenn die Angst vor dem Fremden geschürt wird. An dieser Stelle betreten wir jedoch die Ebene des politischen und militärischen Manipulationspotentials einer Gesellschaft, die nicht Gegenstand dieses Vortrages ist.

Zunächst einige Beispiele, die die Konfliktfelder verdeutlichen: Nehmen wir die Verständigung bei der Annäherung der Geschlechter, wenn diese aus verschiedenen Kulturkreisen kommen und die Tendenz aufweisen sich an den Selbstverständlichkeiten ihres eigenen Kulturkreises zu orientieren. Dies wird häufig an beobachtbaren Verhaltensunterschieden verdeutlicht, was dem Verständnis des Problems anfänglich sehr zuträglich ist. Zum Beispiel hören wir, daß amerikanische Männer in Kontakt mit englischen Frauen sich gegenseitig Mißver- 
ständnisse bei der Annäherung liefern, weil sie sich dabei an bestimmten Spielregeln orientieren, die in diesen Ländern eine andere Reihenfolge haben. Amerikanische Männer sind z. B. gewohnt, einen Kuß als einen eher unverbindlichen Teil eines intensiveren Sich-Kennenlernens zu deuten, während ihre englischen Partnerinnen eher dazu tendieren, ihn als eine wichtige Stufe der Annäherung zu interpretieren. Auch hören wir, daß Eskimos den Mundkuß nicht schätzen, sich dafür aber gerne an ihren Nasen reiben, oder daß die Ehefrau mit dem geschätzten Gast als Zeichen von Annahme und Achtung eine Nacht verbringen soll. Oft hören wir, daß die Jungfräulichkeit einer Frau Voraussetzung für das Interesse eines Mannes oder die Annäherung der Geschlechter eine Familiensache ist, und so erkennen wir weiter, daß letztlich jedes Denksystem (Religion, Gesellschaft, Wissenschaft, Philosophie etc.) beschreibbare Spielregeln für die Annäherung der Geschlechter aufstellt. Wenn wir konsequent fortfahren, sehen wir, daß innerhalb eines Landes, einer Provinz oder einer Gemeinde die Erwartungen einer Familie sich von einer anderen in dieser Hinsicht unterscheiden und bei NichtBeachtung derselben ernsthafte persönliche Verletzungen verursacht werden können. Nicht zuletzt erkennen wir, vor allem wenn wir jemanden näher kennen oder mit ihm zusammenleben, daß er Gewohnheiten pflegt, die sich von unseren so weit unterscheiden, daß wir es manchmal nicht schaffen, diese Unterschiede gewinnbringend zu verarbeiten, und dann an der Inkompatibilität unserer Art und Weise, uns mit unserer Welt in Beziehung zu setzen, scheitern. Spätestens hier schon zeigt das erste vom Watzlawick (1985) aufgestellte metakommunikative Axiom „Man kann nicht nicht kommunizieren“ seine Janusköpfigkeit.

D. h. weiter, daß die Erwartungen und Wege zum Verständnis des „Anderen“ sich bereits innerhalb eines „homogenen“ Kulturkreises voneinander erwartungsgemäß und graduell unterscheiden werden, je nachdem ob wir es mit einem unorthodox denkenden Menschen, einem christlichen Priester, einem Sozial- oder Naturwissenschaftler oder mit dem Anhänger einer philosophischen Richtung zu tun haben. Die Mittel, derer sie sich bedienen, um sich in der Welt zu orientieren bzw. ihren Beruf oder Berufung auszuüben, und die Art, ihr Wissen dafüir zu erlangen, werden unterschiedlich ausfallen, ebenso wie die Wege zur Befriedigung der Erwartungen, die Menschen an sie und sie an sich selbst stellen etc. Die Unterschiede werden vielleicht noch größer sein, wenn wir es mit Vertretern anderer Deutungssysteme zu tun haben wie Medizinmänner, Schamanen, Voodoo-Priester oder sonstige Anhänger bestimmter Kulte magischen Denkens in Kulturen, in denen der Zivilisations- und Aufklärungsprozeß - nach westlichem Verständnis - nicht weit genug vorangeschritten ist. Wenn Vertreter verschiedener Kulturen aufeinander treffen, bedeutet dies nicht gleich, daß sie die jeweilige Deutungsperspektive des anderen nachvollziehen können. Zum Beispiel neigt man in der westlichen Psychologie dazu, den Gedanken nur im metaphorischen Sinn Energie zuzusprechen. Im direkten Gegensatz dazu steht die Ansicht der tibetanischen Buddhisten, daß Gedanken unendliche Kraft besitzen und eigentlich die Materie beherrschen. Je mehr hier einer von dem anderen erfährt, um so mehr bestätigt dies die eigenen Ansichten über den anderen, da ohne eine Änderung der eigenen Perspektive diese nur zur Bestätigung der eigenen Deutung dient.

An diesen Beschreibungen können wir sehen, daß Menschen - auf welcher Ebene auch immer - sowohl gemeinsam wie individuell eine charakteristische und beschreibbare Beziehung zu ihrer Welt herzustellen versuchen, die eng mit ihrer eigenen Entwicklung zusammenhängt. Da wir durch die Aufnahme neuer Informationen unsere Perspektive ändern und weiter entwickeln können, liegt auch hierin die Chance interkultureller Kommunikation, die jedoch von formalen und inhaltlichen Bedingungen abhängig ist. Hier ist kein Platz, um auf diese Bedingungen gebührend einzugehen, weshalb ich nur einige davon erwähne. Wer sich näher dafür interessiert, den verweise ich auf den Forschungsbericht mit den Ergebnissen meines empirischen Forschungsprojektes, das die interkulturelle Kommunikation auf Konflikte hin analysiert hat, um Kriterien für ein „Interkulturelles Übungsprogramm“ auszuarbeiten (Orellana, 1992).

Eine dieser Bedingungen stellt die Bewußtwerdung und Reflexion der eigenen kulturellen Prämissen dar, die in die Kommunikation mit dem Fremden einfließen. Zum Beispiel ist Spontaneität sicherlich eine in allen Kulturen erholsame und sehr gesunde Angelegenheit, die sich jedoch von Kultur zu Kultur unterschiedlich äußert. Deshalb kann sie für die interkulturelle Kommunikation höchstens in Begegnungssituationen hilfreich sein, was den anschließenden Beziehungsaufbau betrifft, ist sie meist jedoch eine Quelle von Mißverständnissen. In diesem Kontext muß die Spontaneität im Kontakt mit fremden Kulturparametern zunächst bewußt gemacht werden und so lange wie nötig durch „Reflexive Intelligenz" ersetzt werden (vgl. Haeberlin/Niklaus, 1978). Dies bedeutet, daß sich die Interagierenden in der Anpassung an den kommunikativen Handlungsverlauf die Möglichkeit bewahren, die eigenen Reaktionen zu verzögern, um diese auf ihre Bedeutung hin zu prüfen, bevor sie unreflektiert eingebracht werden. Spontanes beruht nämlich auf durch Sozialisation selbstverständlich Gewordenem, das allein für eine verläßliche Strukturierung der Kommunikation zwischen Insidern sorgt, die von Menschen anderer kultureller Hintergründe keineswegs geteilt werden muß.

\section{Die Suche nach einem kulturübergreifenden und interkulturell-fähigen Kulturbegriff}

Die Beobachtung bestätigt auch immer wieder, daß interkulturelle Kommunikation u. a. deswegen so schwer ist, weil der Inhalt des Kulturbegriffes diffus konturiert ist. Das Bewußtsein über seine impliziten Bedingungen, Abhängigkeiten, Funktionen und darin enthaltenen Chancen ist unzureichend vorhanden. $\mathrm{Zu}$ oft werden unter Kultur allein künstlerische bzw. geistige Leistungen verstanden, weshalb das eigene Tun kaum im Hinblick auf seine Prämissen reflektiert wird. Deswegen halte ich als eine der Bedingungen gelungener interkultureller Kommunikation auch eine Begriffsklärung für nötig. 
Beim Begriff ,interkulturell“ wird gleich eine Prämisse vorausgesetzt, nämlich daß sich „verschiedene“ Kulturen zueinander in Beziehung setzen und miteinander kommunizieren. Diesem Phänomen möchte ich auf den Grund gehen.

Wenn wir uns auf die Grundsituation menschlichen Daseins besinnen, erkennen wir, daß jeder Mensch, selbstverständlich auch im sozialen Kontext eingebunden, sich selbst als Wahrnehmender seiner Welt erlebt. Somit ist es ihm möglich, eine Interaktion mit der wahrgenommenen Welt bewußt zu gestalten und daher auch - ab einem bestimmten Reifegrad - zu verantworten. Das Ergebnis der steten Interaktion zwischen Mensch und Welt nennen wir Kultur, sie ist daher auch die Vermittlungsinstanz zwischen beiden. So gesehen handelt es sich um die Beschreibung einer Ausgangssituation, welche die Komplexität der Entstehung und Entwicklung von Kultur stark vereinfacht (siehe Abbildung: Grundschema zum Kulturbegriff). Gleichzeitig wird deutlich, daß es gerade diese Einfachheit ist, die je nach Perspektive des Wahrnehmenden, also des Menschen, eine ins Unendliche gehende Vielfalt von Möglichkeiten an Welt- und Menschenbildern zuläßt. So finden wir in jeder menschlichen Kulturform eine beschreibbare Form bzw. ein beschreibbares Ritual wie dort mit dem Tod, mit dem Eintritt ins Erwachsenen Alter, mit der Sexualität etc. umgegangen wird. Diese Formen des Umgangs sind Ergebnis einer aktiven Auseinandersetzung des Menschen mit seiner (Um)Welt, daher sind sie als kulturelle Handlungsfelder zu verstehen, die unsere selektive Wahrnehmung weitgehend bestimmen. Diese selektive Wahrnehmung, so notwendig sie auch ist, um die allägliche Informationsflut in überschaubare Bahnen zu kanalisieren und verarbeiten zu können, ist auch für eine der Hauptschwierigkeiten der Verständigung in der interkulturellen Kommunikation zuständig, nämlich verborgene Bedeutungen eines sichtbaren Verhaltens beim Interagieren miteinander, adäquat zu interpretieren.

Sicher können diese Formen des Umgangs zur Rigidität oder zur Flexibilität hintendieren. Zum Beispiel das Heiratsritual einer Gesellschaft: Wenn in einer bestimmten Kultur das Heiratsritual eine notwendige Bedingung für eine ungestörte Teilnahme am Gesellschaftsleben bedeutet, so daß Menschen, die nicht heiraten, schwere Nachteile im Kauf nehmen müssen (z. B. im Erbrecht oder Arbeitsleben) und dadurch gesellschaftlich stigmatisiert werden, dann könnten wir von einer eher rigiden Form sprechen. Eine eher flexible Form können wir beobachten, wenn das Heiraten für die Individuen noch eine annehmbare Wahlmöglichkeit offen läßt, d. h. wenn die durch das Heiraten entstehenden Nachteile oder Vorteile die soziale Teilnahme nur unwesentlich beein-

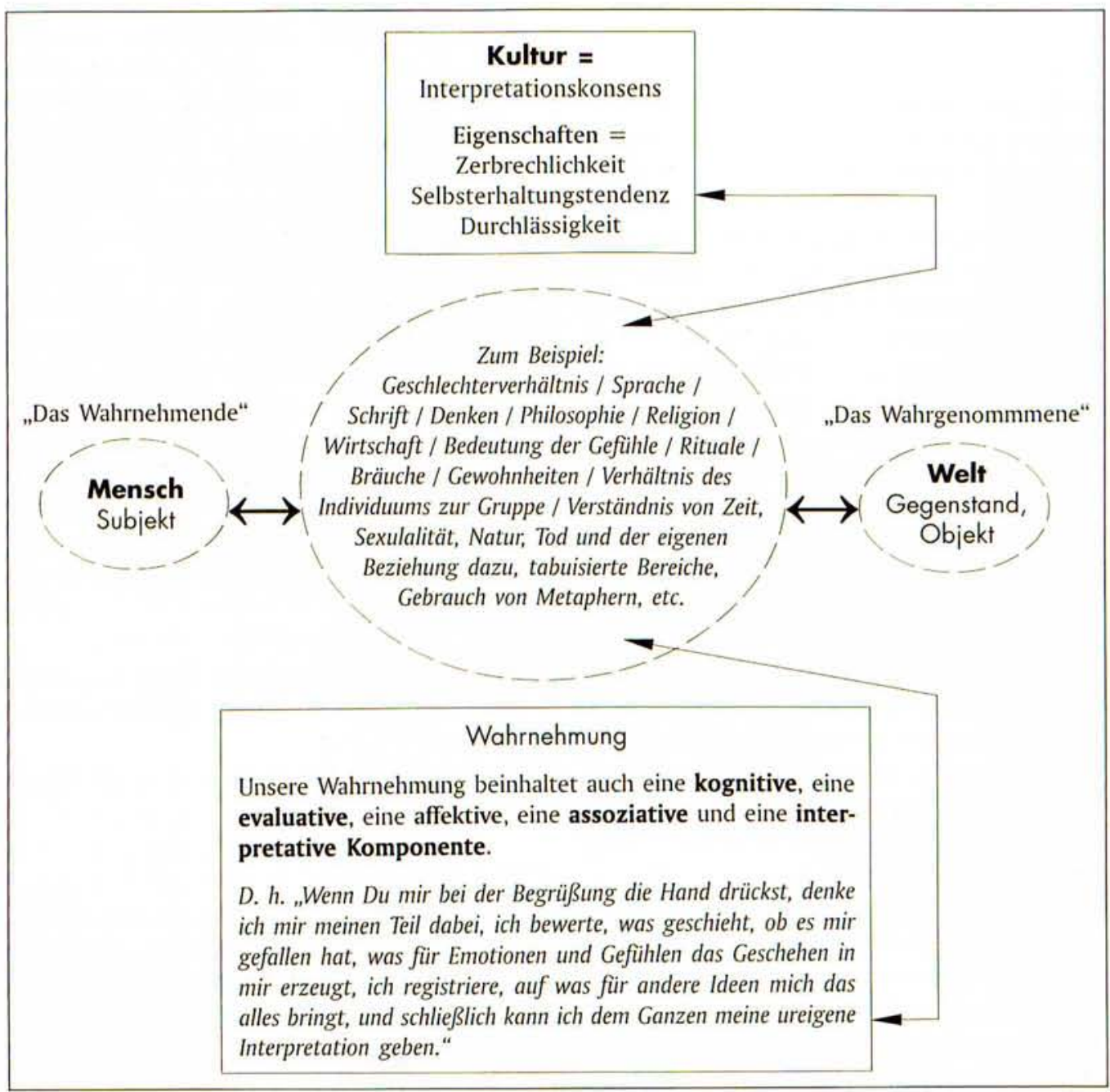

Abb.: Grundschema zum Kulturbegriff 
trächtigen oder fördern. Was die Haltung einer Kultur ihrer Welt gegenuiber angeht, in welcher Form sie sich auch immer manifestieren mag, kann diese eher zur Kooperation oder eher zur Konfrontation hintendieren. Am besten können wir die Umweltproblematik als Beispiel nehmen, um diese kulturellen Tendenzen zu verdeutlichen. Wenn eine Kultur seine natuirlichen Ressourcen über die Maßen ausbeutet und sich dadurch selbst gefährdet, können wir von einer eher konfrontativen bzw. destruktiven Haltung ausgehen. Anders verhält es sich, wenn eine Kultur mit ihren Ressourcen so zu leben lernt, daß diese den Ressourcen die Zeit und die Bedingungen läßt, sich selbst zu regenerieren.

Wenn wir uns in die Lage versetzen wollen bestimmen zu können, was sich wie „verschieden“ zu etwas anderem verhält, sehen wir uns genötigt den Begriff „Kultur“ noch einmal für die interkulturelle Kommunikation als Arbeitshypothese zu definieren:

\section{Der Kulturbegriff}

Aus dem Wörterbuch: Lat. Cultura; Landbau, Pflege/Gesamtheit der geistigen und künstlerischen Lebensäußerungen einer Gemeinschaft.

„Kultur in ihrem weitesten Verständnis weist auf die Form (flexibel oder rigide) und die Haltung (kooperativ oder konfrontativ bzw. destruktiv) hin, durch die eine Gruppe von Menschen mit ihrer phänomenalen Welt eine interaktionale Beziehung herstellt und entwickelt.

Die Bedingung für die Existenz einer Gruppenkultur ist die Bildung eines erfolgreichen, nicht widerspruchsfreien und daher wandelbaren Konsenses, der sich an der Erfüllung wesentlicher, vitaler Funktionen orientiert. Diese Funktionen haben zum Zweck, die Erhaltung der Spezies, die Sicherung des sozialen Zusammenhaltes und die Bildung und Entwicklung der Identität der Kulturteilnehmer zu gewährleisten. Eine weitere organisierende Funktion sorgt für die Regulierung der Struktur, die bestimmt, wer, wann, unter welchen Bedingungen über welche Kulturleistungen verfügen darf.

Die tragenden und/oder bündelnden Handlungsfelder einer Kultur in ihrer Gesamheit (z. B. Religion, Philosophie, Traditionen, Ökonomie, Werte, Sprache etc.) bilden den kulturellen Konsens und sind wie dieser in stetiger Wandlung begriffen. Sie üben für den Menschen die Funktion eines dynamischen Bindegliedes bzw. die eines vermittelnden Elements zwischen ihm und seiner Welt aus, sie schaffen und bestimmen die Deutungs- bzw. Interpretationsweisen derselben."

Die kaum entdeckte Bereicherung, die sich bei erfolgreicher interkultureller Kommunikation offenbart, besteht im wesentlichen in einer allgemeinen qualitativen und quantitativen Steigerung der Bewußtseinsinhalte einer Person, ihrer Lernfähigkeit und ihrer Intelligenz. Die Zunahme an Deutungsperspektiven oder auch Informationsmengen in diesem ,pluralistischen Universum“, wie dies von William James konzipiert wurde, kann die Person zur Verwirrung und zu einer inneren Krise führen, die ihr aber bei erfolgreicher Verarbeitung zu differenzierteren Zugängen zu Informationen jeder Art und somit zu einer breiteren Entscheidungsgrundlage verhilft. Dieser Prozeß beinhaltet auch eine Erweiterung und Vertiefung des eigenen Menschen- und Weltbildes.
Das dabei erworbene Verhaltensrepertoir wird durch diese Kommunikation zunehmend erweitert und soziale Kompetenzen können erhöht werden. Die Folge ist eine Intensivierung vitaler Prozesse, die von der Person als Bereicherung erlebt wird und ihr gleichzeitig als Indikator für psychisches Wohlbefinden dient.

\section{Fußnote}

1 Schriftliche, überarb. Fassung des Vortrages (Gehalten in der TU Berlin am 29.01.1993

\section{Empfohlene Literatur zum Thema}

Haeberlin, U./Niklaus, E. (1978): Identitätskrisen. Theorie und Anwendung am Beispiel des sozialen Aufstiegs durch Bildung. UTB 697, Verlag Paul Haupt Bern und Stuttgart, 1978.

Haumersen, Petra/Liebe, Frank: Studie der Berlin-Forschung zum Thema „Ansätze experimenteller Formen interkulturellen Lernens am Beispiel des internationalen Jugendaustausches“ (HIZ-Kurzmeldung Nr. 1643, 1989). Mehan, Hugh \& Wood, Houston (1976): Fünf Merkmale der Realität. In: Ethnomethodologie. Beiträge zu einer Soziologie des Alltagshandelns. Weingarten, Elmar/ Sack, Fritz/Schenkein, Jim (Hrsg.), Suhrkamp Taschenbuch Wissenschaft 71, Frankfurt a.M., S. $29 \mathrm{ff}$.

Orellana Aguirre, D. (1992): Berlin-Forschung: Psychologische Aspekte zur Entwicklung eines begleitenden interkulturellen Übungsprogramms für Deutsche und Ausländer im Bereich der psychosozialen Fluichtlingsarbeit. Forschungsbericht. Publikation der Berlin-Forschung der Freien Universität Berlin, November 1992. Dort findet der Leser weiterführende Literatur. Der Forschungsbericht ist kostenlos zu beziehen bei der Berlin-Forschung, Altensteinstr. 48, 14195 Berlin, Tel. (030) 8383509

Schütz, Alfred (1972): Der Fremde. Ein sozialpsychologischer Versuch. Gesammelte Aufsätze Bd.II, Studien zur soziologischen Theorie. Martinus Nijhoff/Den Haag. Schütz, Alfred (1972): Der Heimkehrer. Gesammelte Aufsätze Bd. II, Studien zur soziologischen Theorie. Martinus Nijhoff/Den Haag.

Watzlawick, Paul/Beavin, Janet H.Jackson, Don D. (1969): Menschliche Kommunikation. Formen, Störungen, Paradoxien. 7. unveränd. Aufl., Bern/Stuttgart/Wien: Huber, 1985.

Watzlawick, Paul (1977): Die Möglichkeit des Anderssein. Zur Technik der therapeutischen Kommunikation. Verlag Hans Huber Bern, 1977 (dort das Kapitel über Weltbilder, S. 37-43).

\section{Verfasser}

Dipl.-Psych. Daniel Orellana Aguirre

Technische Fachhochschule Wildau

Sprachenzentrum

Bahnhofstraße

15745 Wildau 